\title{
Proceso de formulación y validación de las guías alimentarias para la población chilena
}

\section{Development and validation process of food-based dietary guidelines for the chilean population}

\begin{abstract}
The Food Based Dietary Guidelines (FBDG) are educational tools that help people to select appropriate foods for good nutrition and health. Most countries have FBDG's, but its dissemination and implementation have shown weaknesses. This paper presents the development process and validation of the 2012 FBDG for the Chilean population. The latest scientific evidence was reviewed and new messages were validated with METAPLAN, a qualitative-quantitative method for problem analysis applied to information gathered with 48 focus groups from Arica, Santiago and Chillán-Concepción. The messages promote consumption of healthy foods, focusing on critical nutrients (sugar, saturated fats, sodium) and energy intake reduction. Final version of the messages considered comments and suggestions from participants and from two expert panels. Its implementation should include promotion strategies that involve the Ministry of Health, Education, Agriculture, universities, industry and mass media, in order to achieve changes in feeding behavior in the most vulnerable segments of the Chilean population.

Key words: Food Based Dietary Guidelines (FBDG), healthy foods, critical nutrients, nutrition education.
\end{abstract}

\section{Sonia Olivares C. Isabel Zacarías $H$. Carmen Gloria González G. Elisa Villalobos $V$.}

Instituto de Nutrición y Tecnología de los Alimentos (INTA), Universidad de Chile, Santiago, Chile

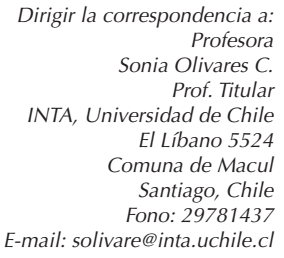

Este trabajo fue recibido el 17 de Abril de 2013 y aceptado para ser publicado el 6 de Julio de 2013.

\section{INTRODUCCIÓN}

La Conferencia Internacional sobre Nutrición (CIN), realizada en Roma por la FAO y la OMS el año 1992, incorporó por primera vez a las enfermedades crónicas no transmisibles (ENT) como un importante problema de salud pública mundial, reconociendo que afectaban con más fuerza y gravedad a los sectores de menor nivel educacional e ingresos. Considerando que los estilos de vida de las personas constituían los principales factores de riesgo, en especial los asociados a sus conductas alimentarias y de actividad física, se recomendó a los gobiernos elaborar, implementar y evaluar Guías Alimentarias Basadas en Alimentos (GABA), remplazando el enfoque existente hasta esa fecha, basado en nutrientes (1). En la Declaración Mundial y Plan de Acción de la CIN, firmados por 159 estados (2), se indicó que cada país debía desarrollar sus propias GABA, de acuerdo a sus principales problemas de salud pública, con mensajes relevantes para personas de distinta edad, cultura y estilos de vida.

El propósito de las GABA es ayudar a la población general a identificar y seguir las recomendaciones alimentarias para una buena nutrición y salud. Constituyen una herramienta fundamental para la educación en nutrición, a ser usada por profesionales de salud, profesores, periodistas, extensionistas y otros profesionales que trabajen directamente con el público.

La Consulta Conjunta FAO/OMS "Preparación y uso de guías alimentarias basadas en alimentos", realizada en Nicosia, Chipre (3), estableció las bases para su desarrollo y uso, con el fin de mejorar los patrones de consumo de alimentos y el bienestar nutricional de individuos y poblaciones. Entre los pasos para el desarrollo de las guías nacionales se incluyó la formación de un equipo de trabajo; la revisión de los patrones de consumo de alimentos y los problemas críticos de salud, alimentación o nutrición que serán el objetivo de las GABA; la definición de propósitos, metas y la población objetivo de las GABA; asegurar que los mensajes sean socioeconómicamente factibles y culturalmente aceptables; validarlos para asegurar su pertinencia y aceptación y realizar el seguimiento de los cambios en la alimentación y el estado nutricional de las personas.

En la reunión organizada por la FAO para conocer los 
avances en la elaboración y uso de las GABA en países de América del Sur en 1998 (4), se encontró que sólo el Ministerio de Salud de Chile había publicado GABA para la población mayor de dos años (5) y Venezuela para los menores de 6 años. Algunos países estaban avanzando en su elaboración y otros aún no habían iniciado el proceso. En las conclusiones se indicó que las GABA debían contener el menor número de mensajes posible y ser validadas con grupos representativos de la población objetivo; establecer un plan para cumplir con los objetivos propuestos, realizar su seguimiento y evaluación y establecer normas éticas para las relaciones entre el gobierno, las universidades y la industria (4).

Considerando que algunos países del Mediterráneo, Medio Oriente y Asia tenían una de las tasas más rápidas de crecimiento de la obesidad en el mundo, la FAO y la OMS realizaron una consulta técnica en El Cairo el año 2004, con la participación de Bahrein, Egipto, Grecia, India, Kuwait y Pakistán (6). Se concluyó que en vista de la doble carga que afectaba a los países de la Región, se debía actuar en forma inmediata en el desarrollo e implementación de GABA, ya que éstas podrían contribuir significativamente a la implementación de la Estrategia Global sobre Dieta, Actividad Física y Salud publicada por la OMS el año 2004 (7).

El año 2005, el Ministerio de Salud de Chile publicó la segunda versión de las guías alimentarias, las que incluyeron mensajes técnicos y comunicacionales. Estas guías formaron parte de la Guía para una Vida Saludable, que incluyó además recomendaciones en actividad física y consumo de tabaco, además de mensajes psicosociales (8).

El año 2007, la OPS/OMS y el INCAP realizaron una Consulta Técnica Regional en Antigua, Guatemala, en la cual se revisó el grado de avance de las GABA en los países de América Latina, identificando que si bien 15 países las habían elaborado, la mayoría había tenido limitaciones para su implementación y sólo 3 habían evaluado parcialmente la etapa de elaboración. Se destacó la escasa experiencia en el monitoreo y evaluación de la fase de implementación (9).

En ese mismo año, el Fondo Mundial para la Investigación del Cáncer (WCRF) y el Instituto Americano para la Investigación del Cáncer (AICR) (10), realizaron una revisión sistemática de la evidencia científica y elaboraron doce recomendaciones para prevenir esta enfermedad, entre las cuales explican cómo las elecciones que cada persona puede hacer con respecto a su alimentación, actividad física y control del peso pueden reducir sus posibilidades de desarrollar cáncer. Estas recomendaciones son: "Elija principalmente alimentos vegetales, limite las carnes rojas y evite las carnes procesadas", "Sea físicamente activo de cualquier manera durante 30 minutos o más cada día" y "Aspire a tener un peso saludable durante toda la vida".

Los EE.UU., uno de los países pioneros en la formulación de guías alimentarias, las que actualiza cada 5 años, ha experimentado interesantes cambios tanto en su enfoque, como en la gráfica que complementa su difusión y educación. En su versión del año 2010, se observa un énfasis en los mensajes referidos al balance energético, el mantenimiento del peso corporal, los alimentos de alta densidad energética y alto contenido de grasas saturadas, azúcar y sodio, de los que sería necesario reducir el consumo y alimentos de los que sería necesario aumentar el consumo en grupos específicos (11).

Las GABA publicadas por el Consejo Nacional de Nutrición de Noruega el año 2011 (12), indican que en la revisión de la literatura se priorizó la evidencia científica basada en alimentos y muchos de los mensajes específicos son cuantitativos. El mensaje general señala: La dieta recomendada es principalmente a base de vegetales, frutas, berries, granos enteros y pescado, limitadas cantidades de carnes rojas, sal, azúcares agregados y productos de alta densidad energética. Se recomienda además realizar al menos 30 minutos al día de actividad física.

Si bien la mayoría de los países de América Latina ya cuentan con GABA, su implementación presenta grandes falencias. Como se reconoció en la consulta técnica de Antigua (9), en general la difusión ha estado centrada en el sector salud y sólo en algunos casos ha contado con el apoyo de educación. Se observa una ausencia de campañas a través de los medios masivos y una baja utilización en las políticas y programas alimentarios.

El objetivo de este estudio es presentar el proceso para la formulación y validación de las nuevas GABA para la población chilena, incluyendo algunas sugerencias para su difusión e implementación.

\section{MATERIAL Y MÉTODOS}

En la figura 1 se presentan las fases del proceso de elaboración de las nuevas GABA para la población chilena.

Fase 1. Revisión de la literatura en la formulación de GABA para la población chilena

El estudio incluyó artículos de actualización sobre la evidencia epidemiológica, la disponibilidad y consumo de alimentos en distintos grupos de la población chilena, un análisis crítico de la literatura respecto a la elaboración, implementación y evaluación de las GABA; una revisión de las publicaciones recientes sobre el balance energético y los nutrientes de los que sería necesario reducir su consumo: grasas, azúcares y sodio. Adicionalmente, se incluyeron breves actualizaciones sobre los nutrientes que presentan déficit en algunos grupos vulnerables, como hierro, zinc, calcio, folatos, vitamina B12 y vitamina D. Adicionalmente se incluyó un artículo sobre fibra dietética.

Estos artículos fueron elaborados por especialistas del INTA, Universidad de Chile, la Universidad de La Frontera y la Universidad de Playa Ancha. Para la revisión de la literatura internacional sobre GABA se contó con el apoyo de una Consultora Internacional de FAO-Roma. La edición estuvo a cargo de las investigadoras responsables (13).

Fase 2. Elaboración preliminar de los mensajes

En una primera instancia, el equipo técnico a cargo del estudio elaboró 29 mensajes técnicos que recogían la evidencia nacional e internacional. Se incluyó la revisión de las guías alimentarias para la población chilena publicadas en 1997 y 2005, y evaluaciones de ellas realizadas con profesionales de salud y escolares de enseñanza básica en tres regiones del país $(5,8,14-16)$.

Después de revisar las últimas versiones de las guías alimentarias de 24 países de los cinco continentes, se consideró en forma especial las últimas Guías Alimentarias de Estados Unidos de Norteamérica (11) y las de Noruega (12), por su enfoque más ajustado a las necesidades de la actual situación epidemiológica de la población chilena.

La primera versión de las Guías técnicas, realizada por el equipo a cargo del estudio y clasificada en 11 sub-temas, fue analizada en un taller con un equipo de expertos constituido por académicos del INTA, Universidad de Chile, la Universidad del BioBio, profesionales del Ministerio de Salud, la Junta Nacional de Auxilio Escolar y Becas (JUNAEB), la Junta Nacional de Jardines Infantiles (JUNJI) y la FAO. 
A estos expertos se les envió la propuesta preliminar de mensajes técnicos ( 3 a 4 opciones para cada sub-tema) antes del taller, con el fin de recibir sus comentarios $y$, a través de ellos, los de otros profesionales de su sector, para lograr consensos más amplios. Durante el taller, se discutieron los mensajes seleccionados por los expertos, los que representaban también la opinión de profesionales y estudiantes de nutrición. Con estos antecedentes, se elaboró una lista de 22 mensajes ( 2 por sub-tema), para ser validados con 48 grupos de la población en tres regiones del país.

Fase 3. Método utilizado para la validación de las guías

El trabajo de campo se realizó a través de un estudio cualicuantitativo, en el que se utilizó el método para el análisis de problemas denominado Metaplán $(17,18)$ con personas sanas de distintos grupos de edad, sexo y nivel socioeconómico (NSE) en tres regiones del país (norte, centro y sur), que participaron en forma voluntaria. Este método consiste en discutir una situación en grupos pequeños (8 a 10 personas), anotando y visualizando las opiniones de todos los participantes, quienes inicialmente anotan sus respuestas con un plumón en una tarjeta con 1 a 3 palabras en letra grande, todas las cuales son expuestas al grupo para la discusión posterior.

Para clasificar el nivel socioeconómico en los adultos y adultos mayores se utilizó la Matriz de Clasificación Socioeconómica ESOMAR (World Association of Mental Research) la cual fue adaptada a la realidad chilena por ADIMARK (19). En el caso de los escolares, se clasificó como NSE medio bajo a los asistentes a escuelas públicas ubicadas en sectores de bajos ingresos y como NSE medio alto a los asistentes a colegios privados pagados, ubicados en sectores de altos ingresos, como se ha hecho en estudios previos (20).

Para el desarrollo de la sesión se diseñó una guía de trabajo, con las instrucciones para el moderador o facilitador. Esta guía incluyó los 22 mensajes y el desarrollo de las etapas en las que se analizaría cada par de mensajes con los participantes.

Las sesiones fueron grabadas y el observador registró los aspectos más relevantes de la reunión en la guía elaborada especialmente para ese fin. Las dos guías fueron diseñadas y probadas en dos sesiones piloto realizadas en Santiago y dos en Arica.

El desarrollo de cada sesión de trabajo se organizó en tres etapas, en base a las instrucciones planteadas por el moderador, las que se detallan a continuación:

1. Entre los dos mensajes que se presentan, por favor anoten en su tarjeta el que cumpla con las siguientes características:

- Llama la atención

- Es fácil de entender

- Es apropiado para usted (o para ti)

Después de agrupar y exponer las respuestas a esta pregunta, el moderador solicitó a los participantes explicar el motivo de su elección y dio espacio para la discusión del grupo.

2. En la segunda tarjeta se solicitó a los participantes que anotaran para quien o quienes creían que estaba dirigido el mensaje; la palabra o palabras que les habían parecido poco claras o difíciles de entender y sus propuestas de cambio.

3. En la tercera tarjeta, se solicitó a los participantes responder lo siguiente:

¿El mensaje seleccionado es fácil o difícil de poner en práctica para usted? ¿Por qué?

Con las opiniones de todos, se trató de llegar a acuerdo sobre lo que los participantes deseaban agregar, quitar o cambiar a los mensajes seleccionados. La duración de cada sesión de Metaplán para el análisis de las Guías Alimentarias demoró entre 80 y 120 minutos. El método tiene la ventaja de lograr la participación de todos y contar con una constancia escrita de lo tratado y acordado en las distintas sesiones, lo que es complementado con las grabaciones de los comentarios generales.

Proceso de elaboración de las GABA para la población chilena.

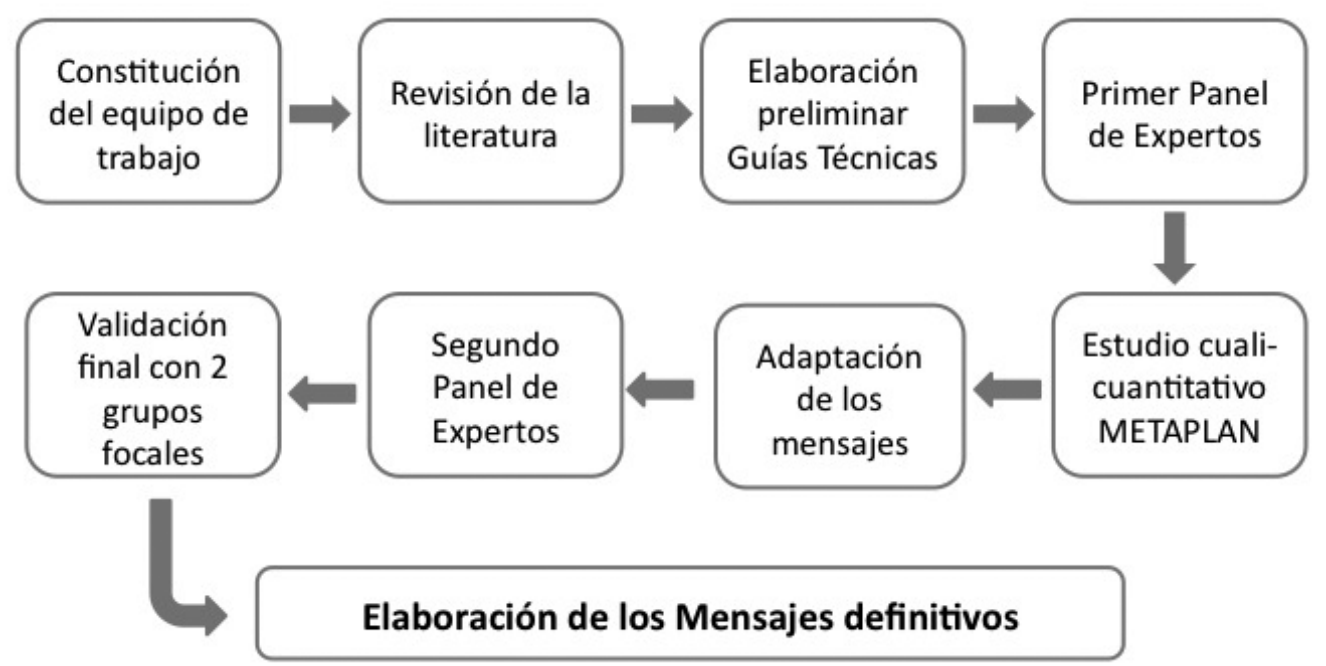


Distribución de los grupos participantes en la validación de las GABA

El trabajo se realizó en Santiago, representando al centro; en una ciudad del norte (Arica) y dos del sur del país (Chillán-Concepción). En cada región se seleccionó y capacitó a profesionales para la realización de las sesiones de Metaplán. En Arica, la moderadora y observadora fueron una profesora de alimentación y una educadora de párvulos; en Santiago y Chillán-Concepción nutricionistas con experiencia en educación en nutrición.

La capacitación de las profesionales que realizaron el trabajo de terreno estuvo a cargo de las investigadoras responsables del estudio y se realizó a través de demostraciones utilizando el Metaplán, con la coordinadoras locales como observadoras. Posteriormente se supervisó el desarrollo de uno o dos grupos a cargo de las profesionales que desarrollarían las actividades en cada ciudad.

La distribución de los grupos de acuerdo a las variables citadas se detalla en la tabla 1.

Cabe destacar que en el grupo de 20 a 60 años se eligió de preferencia a los adultos jóvenes (20 a 35 años), en especial a madres de preescolares, por su importante responsabilidad en la formación de hábitos saludables en sus hijos, lo que también incluyó a los actuales o futuros padres.

Este estudio fue aprobado por el Comité de Ética del INTA.

\section{Elaboración de las GABA definitivas}

Con el análisis de contenido de los resultados de los grupos focales, se modificaron los mensajes de las guías. La versión resultante fue sometida a la revisión y aprobación de un segundo panel de expertos, el que se realizó en dependencias de la Representación de la FAO en Chile. Participaron representantes de los Ministerios de Salud, Agricultura, Edu- cación, JUNAEB, la FAO, OPS/OMS, el INTA y la Escuela de Nutrición de la Universidad de Chile, la Escuela de Nutrición de la Universidad del Bío-Bío, la Escuela de Nutrición de la Universidad Mayor y el Colegio de Nutricionistas de Chile.

En la última etapa del estudio, los mensajes resultantes fueron sometidos a una nueva evaluación con dos grupos de consumidores de Santiago. El primero estuvo constituido por especialistas en comunicaciones y el segundo por técnicos y profesionales de distintas áreas.

\section{RESULTADOS}

De los estudios cuali-cuantitativos de validación de los mensajes

Los 48 grupos focales recogieron la opinión de 380 personas, permitiendo la selección de 11 mensajes, que obtuvieron sobre el $60 \%$ de aprobación en todos los grupos.

En la tabla 2 se presentan los mensajes con las sugerencias de los grupos focales realizados con personas de distinto sexo, edad y NSE en Arica, Santiago y Chillán-Concepción, que fueron revisados por el segundo panel de expertos y posteriormente por dos grupos finales realizados en Santiago (16 personas). Estos mensajes han incorporado las sugerencias de los 396 participantes, en orden a hacerlos más claros, fáciles de entender y llevar a la práctica y útiles para todo público.

Se analizó las preferencias de cada mensaje en el grupo total, comparando por grupo de edad y se registró los comentarios generales de los participantes en todos los grupos. Se seleccionó la edad como el principal indicador, debido a que mostró las mayores diferencias de opinión observada en los trabajos de grupo. El ejemplo más representativo se refiere al mensaje definitivo $N^{\circ} 2$ : "Pasa menos tiempo frente al computador o la tele y camina a paso rápido, mínimo 30 minutos al día". En los dos mensajes originales sobre este tema el 64,6 y $68,4 \%$ de los niños y jóvenes, respectivamente, seleccio-

TABLA 1

Participantes en los grupos focales de validación de los mensajes de las GABA 2013

\begin{tabular}{|c|c|c|c|c|c|}
\hline Edad & Mujer & Hombre & NSE medio alto & NSE bajo & Total \\
\hline \multicolumn{6}{|l|}{ Santiago } \\
\hline 9 a 13 & 1 & 1 & 1 & 1 & 4 \\
\hline 17 a 19 & 1 & 1 & 1 & 1 & 4 \\
\hline 20 a 60 & 1 & 1 & 1 & 1 & 4 \\
\hline Mayor de 60 & 1 & 1 & 1 & 1 & 4 \\
\hline Sub-Total & 4 & 4 & 4 & 4 & 16 \\
\hline \multicolumn{6}{|l|}{ Arica } \\
\hline 9 a 13 & 1 & 1 & 1 & 1 & 4 \\
\hline 17 a 19 & 1 & 1 & 1 & 1 & 4 \\
\hline 20 a 60 & 1 & 1 & 1 & 1 & 4 \\
\hline Mayor de 60 & 1 & 1 & 1 & 1 & 4 \\
\hline Sub-Total & 4 & 4 & 4 & 4 & 16 \\
\hline \multicolumn{6}{|c|}{ Chillán - Concepción } \\
\hline 9 a 13 & 1 & 1 & 1 & 1 & 4 \\
\hline 17 a 19 & 1 & 1 & 1 & 1 & 4 \\
\hline 20 a 60 & 1 & 1 & 1 & 1 & 4 \\
\hline Mayor de 60 & 1 & 1 & 1 & 1 & 4 \\
\hline Sub-Total & 4 & 4 & 4 & 4 & 16 \\
\hline Total general & 12 & 12 & 12 & 12 & 48 \\
\hline
\end{tabular}

NSE: nivel socioeconómico 
naron el mensaje "Limita tu tiempo frente a la pantalla ( $P C$, TV, celulares) y ayuda a los tuyos a moverse en lugar de estar sentados", en tanto el 52,5\% de los adultos y el 69,7\% de los adultos mayores seleccionaron el mensaje "Camina como si fueras apurado al menos 30 minutos al día o 10 minutos tres veces al día". Esto permite apreciar los cambios entre los mensajes técnicos originales y los definitivos, que incorporaron los comentarios y sugerencias de los participantes.

Entre los comentarios que se repitieron en los distintos mensajes evaluados, las personas valoraron que se les explicara el por qué de cada recomendación y los beneficios asociados. También indicaron que les parecía positivo que se dieran alternativas cuando se indicaba disminuir o evitar el consumo de un grupo de alimentos. Indicaron que no les gustaban los mensajes impositivos y preferían los que sugerían opciones de reemplazo.

El grupo de comunicadores sociales participante en la prueba final sugirió pequeños y valiosos cambios a los mensajes aprobados por el Panel de Expertos, los que fueron incorporados y aprobados por el equipo técnico y el grupo focal final. Con ello se cerró el ciclo de validación y adaptación, respetando las opiniones mayoritarias de todos los grupos.

Las nuevas GABA para Chile fueron aprobadas por el Ministerio de Salud en la Norma General Técnica $N^{\circ} 148$, Resolución Exenta N² 260 el 16 de mayo del año 2013 en la ciudad de Santiago de Chile (21).

\section{DISCUSIÓN}

Las Guías Alimentarias Basadas en Alimentos (GABA), son consideradas un aporte esencial al logro de las metas establecidas por la OMS en la Estrategia Mundial sobre Régimen Alimentario, Actividad Física y Salud 2004 (7), que intentan contribuir a que las personas logren un equilibrio energético y un peso normal; limiten la ingesta energética procedente de las grasas, sustituyan las grasas saturadas por insaturadas; traten de eliminar los ácidos grasos trans; aumenten el consumo de frutas y hortalizas, legumbres, cereales integrales y frutos secos; limiten la ingesta de azúcares libres y la ingesta de sal (sodio).

Desde las últimas GABA publicadas por el Ministerio de Salud el año 2005 (5), los estilos de vida de los chilenos han

TABLA 2

Mensajes de las guías alimentarias para la población chilena 2013.

1. Para tener un peso saludable, come sano y realiza actividad física diariamente.

2. Pasa menos tiempo frente al computador o la tele y camina a paso rápido, mínimo 30 minutos al día.

\section{Come alimentos con poca sal y saca el salero de la mesa.}

\section{Si quieres tener un peso saludable, evita el azúcar, dulces, bebidas y jugos} azucarados.

5. Cuida tu corazón evitando las frituras y alimentos con grasa, como cecinas y mayonesa.

6. Come 5 veces verduras y frutas frescas de distintos colores cada día.

7. Para fortalecer tus huesos, consume 3 veces al día lácteos bajos en grasa y azúcar.

8. Para mantener sano tu corazón, come pescado al horno o a la plancha, 2 veces por semana.

9. Consume legumbres al menos dos veces por semana, sin mezclarlas con cecinas.

10. Para mantenerte hidratado, toma 6 a 8 vasos de agua al día.

11. Lee y compara las etiquetas de los alimentos y prefiere los que tengan menos grasas, azúcar y sal (sodio). 
experimentado grandes cambios. Con un mayor poder adquisitivo y acceso a una diversidad de fuentes de información (televisión, Internet y redes sociales), en prácticamente todos los segmentos de la población, los consumidores actuales son más informados y exigentes, aunque su gran exposición al marketing comercial de alimentos podría explicar el aumento en la ingesta de refrescos azucarados, alimentos de alta densidad energética y comidas fuera del hogar observado en los últimos años $(22,23)$.

Los resultados de la revisión realizada por los especialistas muestran la preocupante situación epidemiológica encontrada en la Encuesta Nacional de Salud (ENS) del año 2010 (24), y considerando que la continua tendencia al aumento de las ENT ya significa graves consecuencias para las personas, las familias y la sociedad, además de una enorme carga económica para el estado, la formulación y adecuada difusión de las nuevas GABA representa una contribución para educar a la población y orientar el diseño de los programas de alimentación institucional para los grupos más vulnerables.

Las recomendaciones de la nueva Ley de Composición Nutricional de Alimentos y su Publicidad, publicada el 6 de julio de 2012 en el Diario Oficial de Chile, que indican una reducción en el consumo de energía, grasas saturadas, azúcares y sodio (25), están en línea con las recomendaciones de la OMS (26) y la evidencia científica mundial (7, 10, 27 - 29).

Las nuevas Guías Alimentarias para la población chilena, si bien se basan en las recomendaciones nutricionales vigentes, se centran prioritariamente en los alimentos, destacando el valor de éstos para mantener un peso saludable y una salud óptima, a través de una alimentación que asegure la mantención del balance energético y una ingesta apropiada de nutrientes esenciales en las distintas etapas de la vida. Considerando el actual perfil epidemiológico de la población, tienen un énfasis en los aspectos referidos a la ingesta de energía y nutrientes críticos (21).

La implementación de las GABA 1997 y 2005 es uno de los aspectos más débiles en la experiencia chilena y de otros países. En general se ha basado en actividades de difusión y capacitación realizadas principalmente por el Ministerio de Salud (16), el INTA de la Universidad de Chile $(30,31)$ y otras universidades. No se ha realizado difusión de las GABA a través de los medios masivos de comunicación. Esto es particularmente relevante, si se considera la importancia que ha adquirido la información a través de estos medios y las redes sociales en los distintos segmentos de la población chilena.

Para lograr cambios en las conductas de las personas, las GABA necesitarán de adecuadas estrategias de comunicación y educación para los grupos más vulnerables, con el fin de intentar mejorar sus actuales conductas alimentarias y tener un impacto en su estado nutricional en el mediano plazo.

El Ministerio de Salud, como responsable de esta iniciativa, tiene la prioridad en la definición de las distintas estrategias y se estima indispensable que en esta oportunidad cree una mayor comunicación y colaboración con los Ministerios de Educación, Agricultura, Desarrollo Social, las universidades y las Organizaciones No Gubernamentales (ONGs), para que las integren en sus diversos programas, utilizando las nuevas herramientas tecnológicas de uso habitual en la población actual. La creación de páginas web especiales para la difusión de las GABA, con información complementaria para educar en alimentación, nutrición y actividad física a distintos segmentos de la población, podría representar una gran ayuda en este sentido.

Con la puesta en marcha de la Ley sobre Composición
Nutricional de los Alimentos y su Publicidad (25), y considerando el alto prestigio social que la población con menor nivel educacional atribuye a los alimentos promocionados a través de los medios masivos, la industria, con el apoyo de dichos medios, podría contribuir a crear el mismo valor para los alimentos de los que se recomienda aumentar el consumo, y ofrecer opciones atractivas y factibles para reemplazar a aquellos de los que se recomienda disminuir el consumo, llevando a una nueva forma de alimentarse a la población chilena, que a su vez aumentará la posibilidad de prevenir las enfermedades relacionadas con la alimentación y mejorar la calidad de vida de las personas, familias y la comunidad como un todo.

\section{RESUMEN}

Las Guías Alimentarias Basadas en Alimentos (GABA), son instrumentos educativos que ayudan a la población a seleccionar alimentos apropiados para una buena nutrición y salud. La mayoría de los países tienen GABA, pero su difusión e implementación presentan grandes debilidades. Se presenta el proceso para la formulación y validación de las GABA 2012 para la población chilena. Se revisó la evidencia científica reciente y se validaron los mensajes con el Metaplán, método para el análisis de problemas, con 48 grupos focales en Arica, Santiago y Chillán-Concepción. Los mensajes promueven el consumo de alimentos saludables, un menor consumo de energía y de nutrientes críticos. En los mensajes definitivos se incorporó los comentarios y sugerencias de los participantes y de 2 paneles de expertos. Su implementación debería incorporar estrategias de promoción de los Ministerios de Salud, Educación, Agricultura, universidades, la industria y los medios masivos, para lograr cambios en el comportamiento alimentario de los segmentos más vulnerables de la población chilena.

Palabras clave: Guías alimentarias basadas en alimentos (GABA), alimentos saludables, nutrientes críticos, educación en nutrición.

Agradecimientos: A Carmen Dárdano, Oficial de Nutrición de FAO/Roma; a las coordinadoras de trabajos de grupo en Arica: María Antonieta Olivares y Pamela Vallejos, en Chillán y Concepción: Jacqueline Araneda, María Angélica Mardones, Alejandra Rodríguez y Norma Venegas.

Este estudio fue financiado por el Ministerio de Salud. Santiago, 2012.

\section{BIBLIOGRAFÍA}

1. Food and Agricultural Organization of the United Nations; World Health Organization. International Conference on Nutrition. Final report of the Conference. Rome: FAO/ WHO; 1992.

2. Organización de las Naciones Unidas para la Agricultura y la Alimentación; Organización Mundial de la Salud. Conferencia Internacional sobre Nutrición. Declaración Mundial sobre la Nutrición y Plan de Acción. Roma: FAO/ OMS; 1992

3. World Health Organization; Food and Agricultural Organization of the United Nations. Preparation and use of food-based dietary guidelines. Report of a joint FAO/ WHO consultation Nicosia, Cyprus. Geneva: WHO Nutrition Program; 1996. Disponible en http://www.fao.org/ docrep/x0243e/x0243e00.HTM. Accedido: Abril 2013.

4. Morón C, Calderón T. La elaboración de guías alimentarias basadas en alimentos en países de América Latina. Food 
Nutr Agricult 1998; 24:19-28.

5. Castillo C, Uauy R, Atalah E. Guías de alimentación para la población chilena. Santiago; MINSAL: 1997.

6. Food and Agricultural Organization of the United Nations; World Health Organization. Technical consultation on national food-based dietary guidelines. Egypt: FAO/ WHO; 2004. Disponible en wWw. http://wWw.fao.org/ docrep/010/ai216e/ai216e00.htm Accedido: Abril 2013.

7. Organización Mundial de la Salud. Estrategia Mundial sobre Régimen Alimentario, Actividad Física y Salud. Ginebra: OMS; 2004.

8. Ministerio de Salud. Guías alimentarias. Resolución Exenta $N^{\circ} 459$ que aprueba Norma general No 76. En: MINSAL, INTA/Universidad de Chile; Vida Chile. Guía para una vida saludable. Santiago: MINSAL/INTA/Vida Chile; 2005.

9. Molina V. Guías alimentarias en América Latina. Informe de la consulta técnica regional de las Guías Alimentarias Basadas en Alimentos. OMS/OPS/INCAP. Antigua, Guatemala, 28-30 de mayo de 2007. Anales Venezolanos de Nutrición 2008; 21(1): 31-41.

10. World Cancer Research Fund, American Institute for Cancer Research. Food, nutrition, physical activity and the prevention of cancer: a global perspective. Washington $D C$ : WCRF/AICR; 2007.

11. US Department of Agriculture; US Department of health and Human Services. Dietary guidelines for Americans 2010. Washington DC: USDA, USDHHS; 2010. Disponible en http://www.health.gov/dietaryguidelines/ Accedido: Abril 2013.

12. Consejo Nacional de Nutrición, Departamento Nacional de Salud Pública. Guías alimentarias basadas en alimentos. Oslo, Noruega; 2011

13. Olivares S, Zacarías I. Estudio para la revisión y actualización de las Guías Alimentarias para la población chilena. Santiago: Ministerio de Salud/INTA, Universidad de Chile; 2012. Disponible en http://www.minsal.cl/portal/url/ item/ddeObc471a56a001e040010165012224.pdf Accedido: Abril 2013

14. Yáñez Y, Olivares S, Torres I, Guevara M. Validación de las guías y de la pirámide alimentaria en escolares de $5^{\circ}$ a $8^{\circ}$ básico. Rev Chil Nutr 2000; 27:358-367

15. Domper A, Zacarías I, Olivares S, Hertrampf E. Evaluación de un programa de información al consumidor. Rev Chil Nutr 2003; 30:43-51.

16. Olivares S, Zacarías I, Benavides X, Boj T. Difusión de Guías Alimentarias por los Servicios de Salud. Rev Chil Nutr 2004; 31(S1): 244 (R82).

17. Organización de las Naciones Unidas para la Alimentación y la Agricultura. Manejo de proyectos de alimentación y nutrición en comunidades. Roma: FAO; 1995.

18. Metaplan LLC. Metaplan Basic Techniques. Moderating group discussions using the Metaplan approach. Princeton NJ, USA.

19. ADIMARK. Matriz de clasificación social modificada ESOMAR. Santiago: ADIMARK; 2000. Disponible en http:// www.microweb.cl/idm/documentos/ESOMAR.pdf Accedido: Abril 2013.

20. Olivares S, Bustos N, Lera L, Zelada ME. Estado nutricional, consumo de alimentos y actividad física en escolares mujeres de diferente nivel socioeconómico de Santiago de Chile. Rev Méd Chil 2007; 135: 71-8.

21. Ministerio de Salud. Aprueba Norma General Técnica $N^{\circ}$ 148 sobre Guías Alimentarias para la Población. Resolución Exenta No 260. Santiago: MINSAL; 2013.

22. Crovetto M, Uauy $R$. Evolución del gasto en alimentos procesados en la población del Gran Santiago en los últimos 20 años. Rev Chil Nutr. 2012; 140: 305-12.

23. Olivares S, Lera M, Mardones MA, Araneda J, Bustos $N$, Olivares MA, Colque ME. Promoción de alimentos y preferencias alimentarias en escolares chilenos de diferente nivel socioeconómico. Arch Latinoamer Nutr. 2011;61(2): 163-71.

24. Gobierno de Chile. Ministerio de Salud. Encuesta Nacional de Salud 2009-2010. Santiago: MINSAL; 2003. Disponible en: http://www.minsal.cl/epidemiología Accedido: Abril 2013.

25. Gobierno de Chile. Ministerio de Salud. Ley $N^{\circ} 20.606$ sobre Composición Nutricional de los Alimentos y su Publicidad. Santiago: Diario Oficial de la República de Chile; 6 de julio de 2012.

26. WHO. Diet, nutrition and the prevention of chronic diseases. Report of a Joint WHO/FAO Expert Consultation. Geneva: WHO; (WHO Technical Report Series 916). 2003.

27. FAO. Fats and fatty acids in human nutrition: report of an expert consultation. Rome: FAO; 2010. Disponible en http://www.fao.org/docrep/013/i1953e/i1953e00.pdf Accedido: Abril 2013.

28. Mozzafarian D, Hao T, Rimm EB, Willett W, Hu FB. Changes in diet and lifestyle and long term weight gain development in women and men. N Engl J Med 2011; 364(25): 2392-2404.

29. Cecchini M, Sassi F, Lauer J, Lee Y, Guajardo-Barron V, Chisholm D. Tackling of unhealthy diets, physical inactivity, and obesity: health effects and cost-effectiveness. Lancet 2010; 376: 1775-84.

30. Olivares S, Zacarías I, Andrade M. Educación en alimentación y nutrición para la enseñanza básica. Morón $C$, ed. Santiago: FAO/MINEDUC/INTA; 2003.

31. INTA, Universidad de Chile. Programa de educación al consumidor. Cartillas educativas para preescolares, escolares, adolescentes, adultos mayores. Disponible en http://www. inta.cl/consumidores Accedido: Abril 2013. 